\title{
Anaplastic pleomorphic xanthoastrocytoma with disseminated growth pattern at the time of diagnosis as well as after treatment: case report and review of literature
}

\author{
Meng Zhu ${ }^{1,2}$, Chen Zhang ${ }^{2}$, Kai Zhao ${ }^{3}$, Leilei Wang ${ }^{4}$, Jian Sun², Yugong Feng ${ }^{1}$, Weicheng Yao', Shizhu Yu ${ }^{5}$,
} Cuiyun Sun $^{5}$ and Xuejun Yang ${ }^{2 *}$

\begin{abstract}
Background: Pleomorphic xanthoastrocytoma (PXA) is usually considered a relatively benign and localized entity. However, cases of PXA with anaplastic features have been reported in recent years. Anaplastic pleomorphic xanthoastrocytoma has been added to the 2016 WHO classification of CNS tumors as a distinct entity.

Case presentation: We describe a rare case of PXA with dissemination, both at the time of diagnosis and after treatment. The 20-year-old male presented with signs of high intracranial pressure and sudden-onset transient seizures. Imaging examinations showed diffuse lesions widely distributed in the left hemisphere, and on histopathological examination, he was diagnosed with anaplastic PXA. The patient underwent surgical treatment and adjuvant concurrent chemoradiation. Follow-up MRI revealed early recurrence and distant spread of the tumor.

Conclusions: Anaplastic PXA usually has unique characteristics, including dissemination, early recurrence, and chemoresistance. A strategy based on early diagnosis and aggressive treatment is warranted. However, sufficiently powered studies are required to generate evidence-based guidelines.
\end{abstract}

Keywords: Anaplastic pleomorphic xanthoastrocytoma, Dissemination, Treatment

\section{Background}

Pleomorphic xanthoastrocytoma (PXA), first described by Kappes et al., is a rare tumor accounting for less than $1 \%$ of all astrocytic tumors [1]. PXA predominantly affects young patients and frequently presents with a history of chronic epilepsy. The lesion mainly appears in the cerebral hemispheres, especially the temporal lobe [2]. PXA usually has a benign biological behavior and is typically associated with a good prognosis [2, 3]. However, cases of PXA with anaplastic features have been reported recently [2, 4-7]. Anaplastic PXA, WHO grade III, has been added to the 2016 WHO classification of CNS tumors as a distinct entity [8]. Patients with anaplastic PXA have shorter survival than patients with

\footnotetext{
* Correspondence: ydenny@126.com

${ }^{2}$ Department of Neurosurgery, Tianjin Medical University General Hospital,

Tianjin 300052, People's Republic of China

Full list of author information is available at the end of the article
}

conventional PXA. Here, we report the case of a student who had anaplastic PXA with a disseminated growth pattern, both at the time of diagnosis and after comprehensive therapy.

\section{Case presentation}

A 20-year-old male patient was admitted to the hospital with a history of nausea, vomiting and headache since 2 days, and sudden-onset transient seizure since $2 \mathrm{~h}$. Ten years ago, the patient sustained an epileptic seizure and brain Magnetic Resonance Imaging revealed no abnormalities. The symptoms improved with standard medical treatment. Physical examination at admission was unremarkable. CT-scan revealed an ill-defined lesion in the right temporal lobe. On MRI, the lesion was found to be widely distributed in the temporal, frontal, parietal, occipital, and insular lobes of the left hemisphere. The lesion was hypointense on T1 images, 
hyperintense on T2 and T2Flair images, and a mild localized enhancement on gadolinium (Gd)-enhanced images (Fig. 1a-d). Positron emission tomography-computed tomography (PET-CT) demonstrated intense ${ }^{18} \mathrm{~F}$-fluorodeoxyglucose (FDG) and ${ }^{11} \mathrm{C}$-methionine (MET) uptake in the left temporal lesion, suggesting an aggressive neoplasm (Fig. 1e and f). The area of tumor on MET images exceeded T2Flair, and likely reflected the invasion front of the tumor.

The preoperative diagnosis appeared to be diffuse astrocytoma or a high grade glioma. A standard left frontaltemporal craniotomy was performed using multimodal image fusion guided navigation and intraoperative MRI technology. The boundary of the tumor was not clear and subtotal tumor resection was performed.

Histopathological examination revealed anaplastic PXA (WHO grade III). Tumor cells were pleomorphic and spindle with abundant pink cytoplasm, and were arranged in a fascicular pattern (Fig. 2a). Many cells had a lipid-filled, foamy cytoplasm interspersed between other cells that had bizarre nuclei, occasionally multinucleated (Fig. 2b). Immunohistochemical staining was positive for glial fibrillary acidic portein (GFAP) (Fig. 2c), S100, vimentin. However, there was a negative expression of O-6methylguanine DNA methyltransferase (MGMT), MDR-1, TP53, and CD34. The Ki-67 labeling index was 10.70\% (Fig. 2d). No signs of necrosis were observed.

Postoperative images showed tumor residue that was not amenable to operative removal (Fig. 3a). The patient had no obvious dysfunction and received adjuvant concurrent chemoradiation 20 days after the surgery. Temozolamide (TMZ, $75 \mathrm{mg} / \mathrm{m}^{2}$ ) was administered during radiotherapy, and subsequent chemotherapy

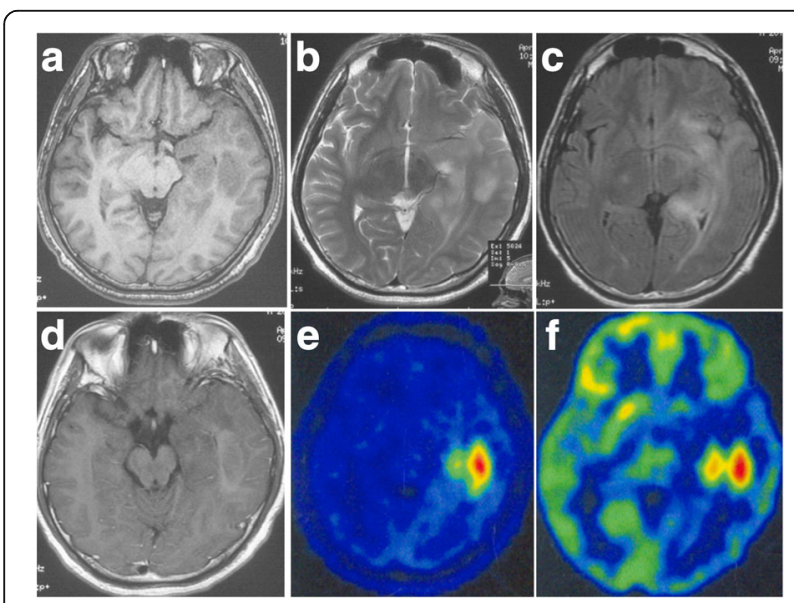

Fig. $1 \mathrm{MRI}$ and PET-CT imaging findings. The lesion was hypointense on T1-weighted image (a), hyperintense on T2-weighted image (b) and T2Flair image (c) with mild localized enhancement on Gd-enhanced image (d). MET-PET showing localized high accumulation in the left temporal lesion (e). FDG-PET showing localized high accumulation in the left temporal lesion $(\mathbf{f})$ cycles of 5 days every 28 days were administered. Three months after surgery, repeat Gd-enhanced MRI showed enhanced lesions around the surgical residual cavity and the left lateral ventricular trigone (Fig. 3b). It was more like pseudoprogression than tumor recurrence. After an observation period of 3 months, the volume of enhanced lesions was found to have increased (Fig. 3c). Therefore, TMZ $\left(150 \mathrm{mg} / \mathrm{m}^{2}\right)$ combined with cisplatin $\left(50 \mathrm{mg} / \mathrm{m}^{2}\right.$ for 2 days) was administered for one cycle. After 6 cycles, MRI showed a decrease in the volume of enhanced lesions, but with concomitant findings of leptomeningeal infiltration around the brainstem and cerebellum (Fig. 3d). Finally, one new enhanced solid lesion appeared in the left frontal lobe at 11 months after the surgery (Fig. 3e). T2Flair images revealed that the tumor cells might even have invaded the opposite hemisphere through white matter fibers (Fig. 3f). The patient died of tumor progression 18 months after the surgery.

\section{Discussion}

PXA is identified as grade II according to the 2007 WHO classification of CNS tumors, and is therefore considered a relatively benign type [9]. Surgical excision is the preferred treatment option and is usually effective. However, some PXAs have been reported to exhibit anaplastic features. The term "PXA with anaplastic features" was proposed in 1999 [2]. A diagnosis of PXA with anaplastic features is usually based on their histological characteristics and the typical molecular characteristics. Giannini et al. [2] proposed criteria for the diagnosis of PXA with anaplastic features, i.e., high mitotic activity (five or more mitoses per $10 \mathrm{HPF}$ ) with or without necrosis. However, many other factors need to be considered in order to standardize the diagnosis and treatment of PXA with anaplastic features. Hirose et al. [10] suggested that necrosis, microvascular proliferation, marked cellular anaplasia, and high Ki-67 index, are also indicative of PXA with anaplastic features. Finally, anaplastic PXA, was added to the 2016 WHO classification of CNS tumors as WHO grade III, instead of the descriptive title of "PXA with anaplastic features". In the current case, Ki-67 labeling index was as high as $10.70 \%$. The patient met the criteria for the diagnosis of anaplastic PXA.

The conventional PXA usually presents as a localized mass which is isointense on T1 images, hyperintense on T2 images, and heterogeneously enhanced. And the mass tends to contain both a solid nodulation and a cystic component. However, anaplastic PXA may manifest very different features on imaging. Different forms of dissemination at the time of diagnosis or after treatment have been documented. Lubansu A et al. [11] reported a 7-year-old girl with anaplastic PXA, with craniospinal meningeal dissemination at the time of diagnosis. 

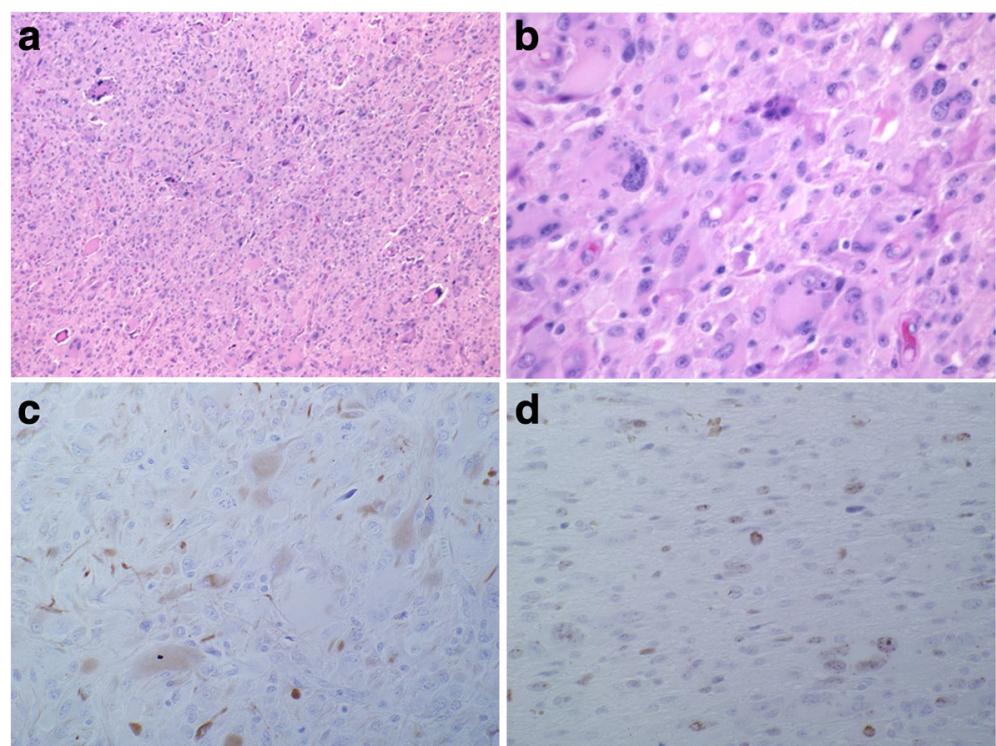

Fig. 2 Photomicrographs of tumor specimens. Photomicrographs of hematoxylin and eosin - stained sections of tumor specimens ( $\times 100)(\mathbf{a})$. Pleomorphic cells with abundant pink, foamy, lipid-filled cytoplasm and multiangular nuclei ( $\times 400)$ (b). Positive expression of GFAP (c). Ki-67 labeling index was $10.7 \%$ (d)

Benjamin et al. [6] reported a case of a 65-year-old man with anaplastic PXA and spinal leptomeningeal spread at the time of diagnosis. In another case, multiple nodular lesions appeared throughout the CNS after surgery in a 47-year-old woman with anaplastic PXA [5]. In our case, the tumor did not present typical radiologic characteristics. There was no cystic component, and tumor dissemination was observed both at the time of diagnosis and after therapy. The tumor invaded multiple lobes and was mistaken for a diffuse astrocytoma prior to the operation. Despite aggressive treatment, the tumor recurred early and spread to distant sites. Whether anaplastic PXA has a stronger ability to invade and disseminate than that of a conventional PXA is a subject of debate. We suggest that PXA with dissemination, early recurrence, or resistance to adjuvant therapy should be treated as anaplastic PXA.

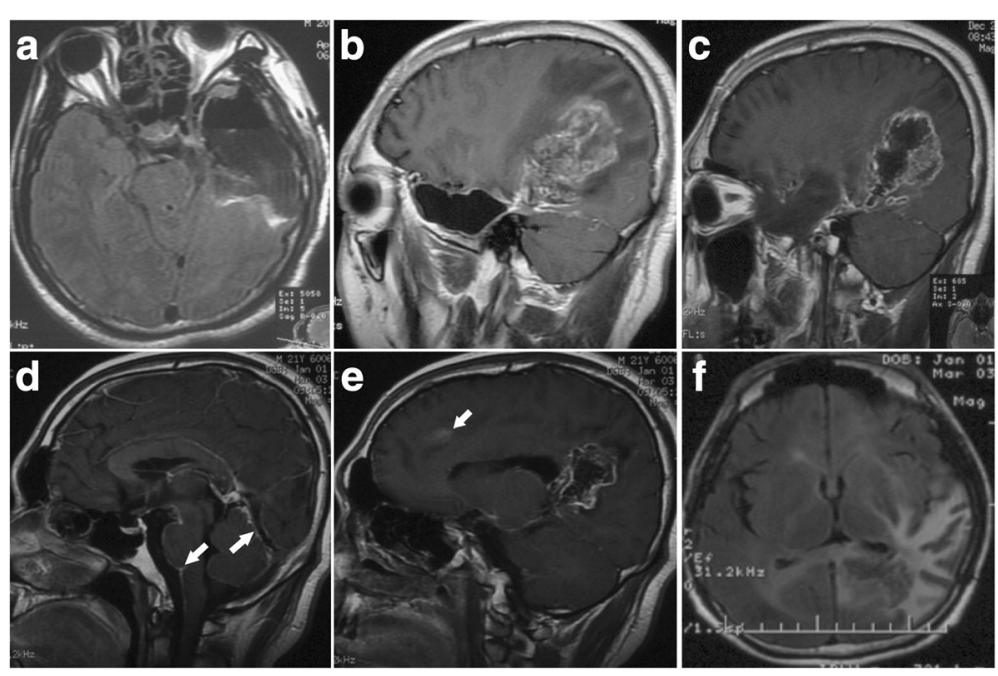

Fig. 3 Follow-up MRI study after the operation. T2Flair image immediately after the operation (a). Gd-enhanced image 3 months after the operation (b). Gd-enhanced image 6 months after the operation (c). Gd-enhanced image 8 months after the operation, arrow: leptomeningeal infiltration (d). Gd-enhanced image 11 months after the operation, arrow: distant spread to the frontal lobe. (e). T2Flair image 12 months after the operation (f) 
A sound understanding of the bio-molecular characteristics is critical to the development of tumor therapeutics. Unlike some low grade gliomas and high grade gliomas, very few researches have focused on the molecular biological features of PXA. Based on the present findings, anaplastic PXA do manifest some distinctive characteristics.

Genetic testing for the BRAF V600E mutation may help differentiate PXA from other astrocytic tumors, since the latter almost always lack this mutation [12, 13]. Previousstudies found the mutation in $17 \%-65 \%$ of anaplastic PXA $[4,14,15]$. However, there is no definitive BRAF V600E mutation status in anaplastic PXA or in conventional PXA. In addition, its clinical value is yet to be established.

It is well known that the efficacy of TMZ is related to the promoter methylation of MGMT [16]. To assess the clinical application value of TMZ for aggressive PXA, G. Marucci et al. [17] examined the MGMT methylation status in 9 PXA patients and 2 anaplastic PXA patients. They found that only 2 PXA patients presented with MGMT promoter methylation. Others, including 2 anaplastic PXA cases, were found to be unmethylated. In another study, Martínez et al. [18] investigated the differences in DNA methylation alterations between conventional PXA and anaplastic PXA. They found that promoter hypermethylation of HOXA5, CD81, ASCL2, HCK and TES genes in anaplastic PXA cases was comparable to that in conventional PXA [18]. However, all these findings need to be confirmed in larger sample based studies to identify clinically relevant biomarkers and guide treatment of anaplastic PXA.

Maximal surgical resection is considered to be the first choice for the treatment of anaplastic PXA. However, the available treatment options following surgical resection have not been adequately investigated. The efficacy of the standard regimen of adjuvant chemotherapy or radiotherapy has not been established on account of the rarity of anaplastic PXA.

A review of published literature reveals that some chemotherapeutic regimens have been used with different degrees of effectiveness, such as vincristine and carboplatin, and TMZ. In our case, we decided to use concurrent TMZ with radiotherapy due to the diffuse growth pattern and the presence of residual tumor. Unfortunately, the tumor recurred and disseminated to distant regions even though TMZ was continuously used and cisplatin was added. Consistent with the results reported by Marucci et al. [17], we also raise doubts about the benefits of TMZ in the treatment of anaplastic PXA. Chamberlain et al. [19] assessed the toxicity and response of vemurafenib, a BRAF inhibitor, in 4 adults with recurrent PXA having BRAF V600E mutation. Vemurafenib appeared to show a single agent activity with acceptable toxicity [19].
However, definitive evidence of its efficacy is yet to be obtained.

\section{Conclusions}

We report a case of anaplastic PXA which is difficult to manage. Although the clinical course of PXA has been acknowledged as being benign, the probability of dissemination, early recurrence, and malignant progression should be noted. Standardized treatment strategy for anaplastic PXA has not been established owing to the rarity of the disease. Based on our summarization, early diagnosis and aggressive treatment appears to be the best strategy in these patients.

\section{Abbreviations}

FDG: 18F-fluorodeoxyglucose; GFAP: glial fibrillary acidic portein; MET: 11Cmethionine; MGMT: O-6-methylguanine DNA methyltransferase; MRI: Magnetic Resonance Imaging; PET-CT: Positron emission tomographycomputed tomography; PXA: Pleomorphic xanthoastrocytoma; TMZ: Temozolamide

\section{Acknowledgements}

Not applicable.

\section{Funding}

We thank grants from the the National Natural Science Foundation of China (no. 81472352) and the Specialized Research Fund for the Doctoral Program of Higher Education (no. 20131202110006). Both of the two fundings were based on the comprehensive therapy of glioma.

\section{Availability of data and materials}

All the data we needed was presented in the main paper.

\section{Authors' contributions \\ $X Y$ and JS conceived the project and designed the study. MZ and CZ collected patient's data. CS, SY, YF and WY provided technical assistance in the study. $K Z$ and LW prepared the illustration. MZ and CZ analyzed data and wrote the paper. All authors approved the paper for the submission.}

Ethics approval and consent to participate

Not applicable.

\section{Consent for publication}

The patient' father provided his kind permission for the publication of this report and any accompanying images, and the written informed consent was provided.

\section{Competing interests}

The authors declare that they have no competing interest.

\section{Author details}

${ }^{1}$ Department of Neurosurgery, Affiliated Hospital of Qingdao University, Shandong 266003, People's Republic of China. 'Department of Neurosurgery, Tianjin Medical University General Hospital, Tianjin 300052, People's Republic of China. ${ }^{3}$ Department of Neurosurgery, Affiliated Hospital of Logistics University of People's Armed Police Force, Tianjin 300052, People's Republic of China. ${ }^{4}$ Department of Neurosurgery, Cangzhou Central Hospital, Hebei 061001, People's Republic of China. ${ }^{5}$ Department of Neuropathology, Tianjin Neurological Institute, Tianjin Medical University General Hospital, Tianjin 300052, People's Republic of China. 
Received: 24 January 2017 Accepted: 19 June 2017

\section{Published online: 08 August 2017}

\section{References}

1. Kepes JJ, Rubinstein L, Eng LF. Pleomorphic xanthoastrocytoma: a distinctive meningocerebral glioma of young subjects with relatively favorable prognosis. A study of 12 cases. Cancer. 1979;44(5):1839-52.

2. Giannini C, et al. Pleomorphic xanthoastrocytoma: what do we really know about it. Cancer. 1999;85(9):2033-45.

3. Fouladi M, et al. Pleomorphic xanthoastrocytoma: favorable outcome after complete surgical resection. Neuro-Oncology. 2001;3(3):184-92.

4. Schmidt $Y$, et al. Anaplastic PXA in adults: case series with clinicopathologic and molecular features. J Neuro-Oncol. 2013;111(1):59-69.

5. Koga T, et al. Long-term control of disseminated pleomorphic xanthoastrocytoma with anaplastic features by means of stereotactic irradiation. Neuro-Oncology. 2009;11(4):446-51.

6. Benjamin C, et al. Anaplastic pleomorphic xanthoastrocytoma with spinal leptomeningeal spread at the time of diagnosis in an adult. J Clin Neurosci. 2015;22(8):1370-3.

7. Okazaki T, et al. Primary anaplastic pleomorphic xanthoastrocytoma with widespread neuroaxis dissemination at diagnosis-a pediatric case report and review of the literature. J Neuro-Oncol. 2009;94(3):431-7.

8. Louis DN, et al. The 2016 World Health Organization classification of tumors of the central nervous system: a summary. Acta Neuropathol. 2016;131(6): 803-20.

9. Louis DN, et al. The 2007 WHO classification of tumours of the central nervous system. Acta Neuropathol. 2007;114(2):97-109.

10. Hirose T, et al. Pleomorphic xanthoastrocytoma: a comparative pathological study between conventional and anaplastic types. Histopathology. 2008; 52(2):183-93.

11. Lubansu A, et al. Cerebral anaplastic pleomorphic xanthoastrocytoma with meningeal dissemination at first presentation. Childs Nerv Syst. 2004;20(2):119-22

12. Bayindir C, et al. Anaplastic pleomorphic xanthoastrocytoma. Childs Nerv Syst. 1997; 13(1):50-6.

13. Dougherty MJ, et al. Activating mutations in BRAF characterize a spectrum of pediatric low-grade gliomas. Neuro-Oncology. 2010;12(7):621-30.

14. Schindler $\mathrm{G}$, et al. Analysis of BRAF V600E mutation in 1,320 nervous system tumors reveals high mutation frequencies in pleomorphic xanthoastrocytoma, ganglioglioma and extra-cerebellar pilocytic astrocytoma. Acta Neuropathol. 2011;121(3):397-05.

15. Dias-Santagata D, et al. BRAF V600E mutations are common in pleomorphic xanthoastrocytoma: diagnostic and therapeutic implications. PLoS One. 2011;6(3):e17948.

16. Hegi ME, et al. MGMT gene silencing and benefit from temozolomide in glioblastoma. N Engl J Med. 2005;352(10):997-1003.

17. Marucci G, Morandi L. Assessment of MGMT promoter methylation status in pleomorphic xanthoastrocytoma. J Neuro-Oncol. 2011;105(2):397-400.

18. Martinez $\mathrm{R}$, et al. DNA methylation alterations in grade II- and anaplastic pleomorphic xanthoastrocytoma. BMC Cancer. 2014;14:213.

19. Chamberlain MC. Salvage therapy with BRAF inhibitors for recurrent pleomorphic xanthoastrocytoma: a retrospective case series. J Neuro-Oncol. 2013;114(2):237-40.

\section{Submit your next manuscript to BioMed Central and we will help you at every step:}

- We accept pre-submission inquiries

- Our selector tool helps you to find the most relevant journal

- We provide round the clock customer support

- Convenient online submission

- Thorough peer review

- Inclusion in PubMed and all major indexing services

- Maximum visibility for your research

Submit your manuscript at www.biomedcentral.com/submit
Biomed Central 